Cahiers d'études italiennes

\title{
La prima pietra tirata. Religion et politique chez Erri De Luca
}

Jean-Claude Zancarini

\section{OpenEdition}

Journals

Édition électronique

URL : http://journals.openedition.org/cei/202

DOI : $10.4000 /$ cei.202

ISSN : 2260-779X

Éditeur

UGA Éditions/Université Grenoble Alpes

Édition imprimée

Date de publication : 15 juillet 2009

Pagination : 145-154

ISBN : $978-2-84310-145-8$

ISSN : $1770-9571$

\section{Référence électronique}

Jean-Claude Zancarini, « La prima pietra tirata. Religion et politique chez Erri De Luca », Cahiers d'études italiennes [En ligne], 9 | 2009, mis en ligne le 15 janvier 2011, consulté le 27 mars 2021. URL http://journals.openedition.org/cei/202 ; DOI : https://doi.org/10.4000/cei.202 


\title{
LA PRIMA PIETRA TIRATA. \\ RELIGION ET POLITIQUE CHEZ ERRI DE LUCA
}

\author{
Jean-Claude Zancarini \\ ENS LSH, UMR Triangle
}

Dans Aceto arcobaleno, le personnage qui, le premier, vient raconter son histoire de violence et de sang au narrateur utilise une expression évangélique pour nommer le destin qui lui est échu. Il déclare que son sort était joué dès le jet de la première pierre : "nella mia prima pietra tirata era già incluso l'uccidere e l'essere ucciso ${ }^{\mathrm{I}}$. " Question brûlante pour une génération, celle qui, lors des années 1968, à vingt ans, pensa la politique en termes d'affrontement et estima qu'il était légitime de dire sa colère et son désir de changement en lançant des pavés ou des sampietrini. Personne alors ne pensait à la phrase du Christ devant la femme adultère « que celui de vous qui est sans péché lui jette la première pierre ${ }^{2}$ ». Quel sens donner à cette traduction dans les mots du fils de l'homme de ce qui était alors pensé dans les termes de la politique radicale ? Erri De Luca veut-il laisser entendre qu'il y eut dans cette génération, dans sa génération, la présomption d'être sans péché qui permet de jeter la première pierre, présomption que n'eurent pas les scribes et les Pharisiens devant la femme adultère ? Le passage à la lutte armée serait alors la conséquence nécessaire de cette présomption, l'acte qui en découle - le jet de la première pierre - contenant en germe la virtualité de la mort donnée ou reçue.

La question de la violence et des rapports qu'elle entretient avec l'aspiration à la révolution est fréquemment posée par Erri De Luca. Il précise son sens dans un passage de Lettera da una città bruciata: la violence n'est pas une fin, le révolutionnaire ne se définit pas par son usage; la violence est une nécessité qui naît de la volonté de résister, de faire durer

I. E. De Luca, Aceto Arcobaleno, Milano, Feltrinelli, I992, p. 32.

2. Jean, 8,7 . 
une communauté - à laquelle il donne, en d'autres passages, le nom de communisme vécu. Il s'agit donc, non d'une violence politique que seul l'État déploie, mais d'une « contreforce », d'une « force de résistance ». Cette contreforce, cette force de résistance, cette violence qui devait exprimer une "volonté de vérité et de justice » Erri De Luca estime que lui et ses camarades l'ont transformée en "volonté de puissance " ${ }^{3}$.

Cette lecture d'un moment historique se fait l'écho de débats de fond qui traversaient la gauche radicale des années I968; celle-ci affirmait, sur la base des thèses marxistes, que la violence est l'accoucheuse de l'histoire, mais n'estimait pas forcément que le moment de la lutte armée était à l'ordre du jour. Ainsi, Lotta continua, le groupe dans lequel Erri De Luca militait, établissait-elle une distinction entre violence de masse et violence d'avant-garde et tendait-elle à se méfier de cette dernière, au nom du lien nécessaire avec le mouvement social. Cette difficulté théorique se résolvait alors dans les termes léninistes de l'analyse concrète d'une situation concrète : en telle ou telle circonstance est-il « juste " (entendre "politiquement efficace ») de prendre les armes ? Erri De Luca semble y donner une réponse globale avec sa phrase sur la transformation collective de la volonté de vérité et de justice en "volonté de puissance ». Cette volonté de puissance pourrait être le nom laïc de la présomption de se croire sans péché, une façon de laisser de côté l'analyse politique et d'y substituer des caractéristiques morales. Notons que cette substitution de la morale à l'analyse politique peut avoir une conséquence importante dans la façon de donner un sens au moment historique considéré, façon qui peut d'ailleurs se lire en filigrane dans la phrase "nella mia prima pietra tirata era già incluso l'uccidere e l'essere ucciso ». On pourrait en effet voir dans cette phrase, au-delà de la trajectoire individuelle du personnage qui la prononce, une parabole pour une génération entière, les actes de révoltes et de refus ayant pour conséquence directe le choix de (ou les dérives vers) la lutte armée. J'ai déjà eu l'occasion d'écrire que cette vision téléologique des événements me paraissait fausse et que précisément il n’y avait pas de lien nécessaire entre le mouvement, fût-il violent dans ses actes et dans ses attendus théoriques, de contestation de l'autorité et le développement de

3. E. De Luca, Lettere da una città bruciata, Napoli, Libreria Dante \& Descartes, 2002, p. 94-96 ; je cite une partie de ce passage, p. $96:$ : [...] la controforza non è rivoluzionaria, è solo una penosa e faticosa necessità di praticare esempi di resistenza. [...] La nostra forza di resistenza non era un valore in sé, non fondava una comunità, cercava solo di farla durare. Era la matrice di un volantino, non le parole scritte sopra. Ridotta a questi termini posso finalmente dire che non l'abbiamo saputa usare. Abbiamo trasformato la nostra volontà di verità $e$ di giustizia in una volontà di potenza." 
la lutte armée 4 . La lecture morale des événements historiques, dont je ne récuse pas la valeur, ne doit pas faire l'impasse sur l'analyse politique des forces en présence et de leurs initiatives : ni la présomption de pureté ni la volonté de puissance ne peuvent expliquer à elles seules le choix massif de la lutte armée dans l'Italie des années d'après 1968.

Notons un autre point, indiqué par l'usage de la métaphore du volantino pour définir la force de résistance (i.e. la violence) de sa génération : "Era la matrice di un volantino, non le parole scritte sopra." La violence comme stencil donc, comme substrat nécessaire pour que des paroles puissent y faire leur trou, cela au sens strict, puis, ronéotypées et distribuées, aller de l'un et de l'une à l'autre, faire sens, faire communauté, le cas échéant communisme. Bien qu'un peu datée et peut-être difficile à comprendre pour qui n'a jamais tourné la manivelle d'une ronéo, la métaphore est belle et efficace. Elle met en évidence un point fondamental : au-delà de la violence (et de toute "techniques»), ce sont bien les paroles qui comptent, celles qui peuvent donner sens à l'interrogation morale sur le sens d'une expérience que De Luca partage avec des milliers de garçons et de filles de sa génération. Ce qui se dit dans cette métaphore est bien la nécessité de l'étude et peut-être de l'écriture.

Dans In alto a sinistra la question du livre, de l'étude et de la filiation est centrale. Le titre du livre désigne à la fois la page suivante, la lecture qui se poursuit " en haut à gauche " et aussi, comme le rappelle l'auteur, la possibilité que quelque part existe "un'antica uscita di emergenza", une sortie de secours, une issue pour le questionnement. Commençons par l'héritage du père : les livres que le père laisse à son fils sont le seul lieu du sens possible à donner à l'expérience vécue, le seul lieu où l'on peut trouver les paroles qui donnent ce sens ("l'unico posto dove l'esperienza che uno fa nel mondo trova le parole d'accompagnamento »), le seul lieu où le passé ("l'angelo del tempo trascorso") se lit lui-même en regardant par dessus l'épaule de celui qui écrit ${ }^{6}$. La question du livre et de l'étude est liée à la question du père, comme dans la religion hébraïque ; et on ne s'étonnera

4. Dans deux articles écrits en collaboration avec Jean-Louis Fournel : « Des historiens peu prudents. L'enjeu historiographique de l'affaire Bompressi, Pietrostefani, Sofri ", Les Temps modernes, n 596 , novembre-décembre 1997 et "Une histoire italienne », Laboratoire italien. Politique et société, nº 2, 200I, p. I27-I38.

5. E. De Luca, Lettere da una città bruciata, op. cit., p. 96 : "[...] intendo ridurre la nostra fitta capacità di contrasto militare a una tecnica."

6. E. De Luca, In alto a sinistra, Milano, Universale economica Feltrinelli, 2005 [I ${ }^{\text {re }}$ éd. I994], p. I23-I24 : "I libri sono il sempre. Chi li scrive può credere di lasciarli ai contemporanei, ai posteri, ma mentre scrive tutto il passato è dietro le sue spalle a leggere. Se non c’è questo angelo del secolo trascorso, se non c’è il suo artiglio sul collo del poeta, le sue parole sono subito cenere. Se non si scrive per essere letto dagli antenati, non resta impresso niente sulla pagina." 
pas de voir apparaittre, derrière le père, la présence de Dieu. Car Dieu a écrit, comme l'Exode en fait foi ("duas tabulas testimonii lapideas, scriptas digito de $\left.i^{7} »\right)$, et Erri De Luca nous dit avoir reconnu cette empreinte du doigt de Dieu, non dans la pierre des tables de la Loi mais dans les vies de certains catholiques qu'il a cotoyés (dont ses amis de la Caritas, avec lesquels il convoyait de l'aide en Bosnie au cours de la guerre en ex-Yougoslavie). Pour dire ce témoignage indirect, il utilise l'expression "impronta digitale ». Dans In alto a sinistra (1994), cette empreinte du doigt de Dieu dans les vies est nettement comparée à celle que l'on peut déceler dans les textes sacrés : "In alcune vite di quelle persone ho visto l'impronta digitale di Dio, cosi come resta nei libri sacri del loro credo ${ }^{8}$. "Il y revient dans Nocciolo d'oliva (2002), dans une phrase qui met en parallèle les paroles de l'Ancien Testament et les vies de ses amis catholiques : "Sono un testimone indiretto: vedo le parole dell'Antico testamento non riducibili a opera di autori vari, vedo le vite degli amici cattolici non riducibili a una buona loro indole o volontà ma scavate da un'impronta digitale. "C'est dans ce même passage qu'il affirme ne pas pouvoir aller au-delà de ce témoignage indirect et ne pas croire en Dieu car il ne sait ni prier ni pardonner?.

Sans doute les premières lectures bibliques d'Erri De Luca visaient-elles à trouver dans les textes sacrés des analogies, des comparaisons avec son expérience vécue ; on peut trouver des exemples de ces lectures " analogiques" dans Una nuvola come tappeto (I99I), lorsque la tentative d'ériger la tour de Babel est présentée comme l'archétype des actions collectives à venir ${ }^{\text {Io }}$ ou lorsque la distribution de la manne céleste au cours de l'exode est définie comme une expression du "socialisme divin » qui servira de

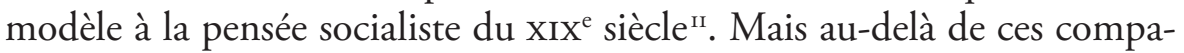
raisons qui sont aussi des clins d'œil, le sérieux de l'engagement dans la lecture et la traduction des textes sacrés, le caractère nécessaire que prennent l'une et l'autre, le noyau d'olive que l'on tourne dans sa bouche, tout

7. Exode, 3I, I8.

8. In alto a sinistra, p. I24.

9. "Con tutto questo rimango uno che parla di Dio in terza persona. Il mio piede urta ogni giorno in questa pietra della preghiera, non la può scavalcare perché la preghiera è la soglia. L'altro inciampo è il perdono. Non so perdonare e non posso ammettere di essere perdonato. " (Nocciolo d'oliva, Padova, Edizioni Messaggero di sant'Antonio, 2002, p. 7)

Io. E. De Luca, Una nuvola come tappeto, Milano, Universale economica Feltrinelli, 2004, [I $\mathrm{I}^{\mathrm{re}}$ éd. I99I], p. I5 : "La macchina-torre che accampava il cielo è il simbolo araldico segreto che sta nel risvolto di bandiera di ogni impresa comune, perché almeno una volta nella vita una persona viene a trovarsi iscritta insieme ad altre nell'ombra di una torre, prima che divenga Babele e disperda i suoi membri." "

II. Ibidem, p. 92 : "Da qui, dal verso diciotto, capitolo sedici del libro dell'Esodo, il socialismo mutuerà la regola morale del fornire a tutti l'indispensabile "; p. 93 : "Il socialismo divino era efficiente, le razioni abbondanti." 
cela me semble à mettre en rapport avec le questionnement moral mis en évidence plus haut. Le rapport au sacré, aux livres puis au Livre, a pour fonction de trouver les paroles qui donnent sens à une expérience ineffable, inouïe : cet "étrange bonheur ${ }^{12}$ " qu'a fait naître pour une génération le communisme vécu " entre un coucher de soleil et une aube». Dans Annuncio mai spedito, un des récits de Il contrario di uno (2003), Erri De Luca raconte la solidarité active des habitants du quartier populaire de la Garbatella avec "la meglio zoventù della città di Roma » attaquée par la police au cours d'une manifestation contre l'OTAN :

Quella gente fa il suo ordine pubblico mettendosi con la meglio zoventù e facendola felice. Perché felicità per noi è stato un quartiere insorto all'improvviso a fianco e intorno. Chiamavamo quelle cose comunismo, ma tiravamo ad indovinare, quella era soprattutto una felicità aspra, affumicata ${ }^{13}$.

Dans un texte antérieur, publié dans Lettere da una città bruciata, il avait déjà fait allusion à la Garbatella insurgée. Il revendiquait le nom de communisme pour ce moment exceptionnel :

Per una notte quel territorio era la libertà ottenuta, il comunismo avuto tra un tramonto e un'alba. Non ci credi, non posso chiamarla così una notte di fuochi e di lampadine accese? Dammi torto, non l'ho capito mai il comunismo se non era quella comunità coinvolta, capace di contagio, di suscitare affetto e furia nel popolo d'intorno, che produceva gesti non chiamati, non voluti, solo fraternità brusca, efficace e sciolta subito al mattino, con un saluto indelebile. Ne avremmo avuti ancora di comunismi lunghi una notte intera ${ }^{14}$.

Cette interrogation sur la façon dont il faut nommer ce qui s'est passé - il est certain que ce fut un bonheur, mais cet étrange bonheur fut-il réalisation du communisme ? - se dit autrement encore : peut-on trouver " une occupation qui ne semble pas privée de sens " quand on a vécu à vingt ans les moments les plus importants de sa vie ? Cette question - qu'il commente abruptement et sans hésitation : "è difficile »-il la pose dans un texte intitulé Fare il mestiere, également publié dans Lettere da una città bruciata ${ }^{\mathrm{IS}}$. Il faut faire un détour, au moins apparent, par cette

I2. "[...] quella strana felicità ", Annuncio mai spedito, in E. De Luca, Il contrario di uno, Milano, Feltrinelli, 2003, p. 55 ; l'expression est également utilisée dans Una storia di strada in Lettere da una città bruciata, op. cit., p. I4.

13. Ibidem, p. 54 .

14. Lettere ad Angelo Bolaffi sull'anno sessantottesimo del millenovecento [1998], in Lettere da una città bruciata, op. cit., p. 32.

I5. "[...] su scala molto ridotta rientro in quella considerazione che Hanna Krall fa a proposito di Marek Edelman, uno dei comandanti della Resistenza nel ghetto di Varsavia. Lei scrive in margine a una intervista con lui: "Quando si vivono a vent'anni i momenti più importanti della propria vita è diffcile trovare un'occupazione 
expérience de vie qu'Erri De Luca nomme "gli anni di madrevita operaia di uno che nacque in borghesia ${ }^{16} "$.

Le choix d'aller travailler comme ouvrier lorsqu'on est un intellectuel n'a pas été seulement un choix individuel; il y a eu un mouvement vers les usines, théorisé en particulier par les militants se réclamant du maoïsme et appliquant les indications contenues dans l'intervention de Mao-Tsé-Toung à la conférence nationale du Parti communiste chinois sur le travail de propagande du I3 mars 1957 qui invitait les intellectuels à partager " deux ou trois ans voire plus " la vie des "masses ouvrières et paysannes ». En France, du fait de la traduction française de ce discours qui parlait de "s'établir », ce mouvement s'est appelé " établissement ". Dans les débats de l'époque et par la suite dans les travaux universitaires qui ont pris ce mouvement d'établissement pour objet la question s'est posée du sens de cet engagement : s'agissait-il d'une décision essentiellement politique, visant à faire du " travail d'organisation dans la classe ouvrière » ou bien fallait-il voir essentiellement dans ce choix une volonté de transformation de l'identité intime des intellectuels, "une expérience de réforme individuelle ». Les deux citations qui précèdent et synthétisent deux façons opposées de concevoir l'établissement sont tirées du livre de Robert Linhart, L'établi, publié en 1978. Robert Linhart, l'un des fondateurs d'un des groupes d'extrême-gauche de l'avant 68, l'Union des jeunesses communistes marxistes-léninistes, fut l'un des promoteurs en France du mouvement d'établissement et fut lui-même établi à l'usine d'automobile de Citroën-Choisy de septembre i968 à juillet 1969. Dans son livre, il revendique clairement la première attitude :

Je ne suis pas entré chez Citroën pour fabriquer des voitures mais pour faire du travail d'organisation dans la classe ouvrière, pour contribuer à la résistance, aux luttes, à la révolution. Dans nos débats d'étudiants, je me suis toujours opposé à ceux qui concevaient l'établissement comme une expérience de réforme individuelle : pour moi, l'embauche d'intellectuels n'a de sens que politique ${ }^{17}$.

Sa conception de l'établissement n'était cependant pas la seule et d'autres établis revendiquaient sans doute " la réforme individuelle " voire la nécessaire rééducation des intellectuels par les masses. C'est cet aspect que souligne (unilatéralement, me semble-t-il) Marnix Dressen, qui a tiré deux

che non sembri priva di senso. È difficile". " (Fare il mestiere [I996], in Lettere da una città bruciata, op. cit., p. 47-48)

16. In alto a sinistra, op. cit., quarta di copertina. L'hapax madrevita indique sans doute un lien possible entre la vie et la langue.

17. R. Linhart, L'établi, Paris, Minuit, 1978, p. 60. 
ouvrages de sa thèse de doctorat sur les établis ${ }^{18}$ : il insiste sur le don de soi, le sacrifice, la foi des établis, leur volonté d'expier la situation de domination de la classe ouvrière. Il applique des postulats théoriques fondés sur l'anthropologie religieuse et sur l'hypothèse d'une " religion sécularisée " qu'il emprunte à Raymond Aron. Cette façon d'analyser - qui minimise l'aspect politique revendiqué par une partie des établis et dont Linhart se faisait le porte parole - met donc l'accent sur le caractère d'emblée religieux de cette posture. Qu'en est-il du parcours ouvrier d'Erri De Luca?

Erri De Luca périodise lui-même assez nettement son expérience de vie ouvrière. S'il se définit comme "uno che ha passato il frattempo tra il '77 e il '9s a fare mestiere di operaio' "', il explicite qu'il faut distinguer les premières années "che rientrano ancora in una specie di scelta" et la suite ; il précise d'ailleurs que ces premières années commencent après la dissolution de Lotta continua en 1976 et que le moment après lequel il ne s'agit plus de faire une vie d'ouvrier par seul choix politique est l'automne I980, "dopo le notti passate davanti al cancello undici di Mirafiori a bloccare a oltranza la fabbrica d'Italia ${ }^{20}$ ". Dans Aceto arcobaleno, le personnage qui se présente comme s'étant enrôlé dans la lutte secrète et ayant tué des hommes explique qu'il a choisi de continuer à mener une vie d'ouvrier après les années de politique, ce "fare convulso » qui contenait en lui les germes d'un " monde futur ${ }^{2 \mathrm{II}}$ :

Poi la gente fu stanca di urti e d'improvviso non ci fu altro da aggiungere. Le migliaia che fummo si sparsero prima a gruppi, poi a polvere. Chi tornò alle professioni di prima, chi agli studi sospesi, mentre altri si sfondavano le vene o si arruolavano in guerre clandestine, io fui tra questi. Sono rimasto a fare l'operaio, là dove avevo cominciato. Mi spinge a questo mestiere solo il desiderio di restare a farlo fino all'ultimo giorno ${ }^{22}$.

La fiction amène à faire le lien entre choix de la lutte armée et choix de la vie ouvrière ${ }^{23}$, mais ce qui m'intéresse ici est la confirmation d'un choix de vie amené par une transformation de la qualité des temps : devenir

I8. M. Dressen, De l'amphi à l'établi. Les étudiants maoistes à l'usine (1967-1989), Paris, Belin, 2000 et Les établis, la chaîne et le syndicat. Évolution des pratiques, mythes et croyances d'une population d'établis maoïstes (1968-1982). Monographie d'une usine lyonnaise, Paris, L'Harmattan, 2000.

19. Lettera da una città bruciata, op. cit., p. 77.

20. Ibidem, p. 47-48.

2I. Aceto arcobaleno, op. cit., p. 20.

22. Ibidem, p. 2I.

23. Pour être précis, le personnage devient ouvrier après avoir quitté le lycée et il est ouvrier pendant ses "sept années de politique " (p. 19-20); il déclare être resté à l'usine après le brusque repli qu'il décrit (p. 2I), puis abandonne le travail salarié lorsqu'il passe dans la clandestinité (p. 35) ; après l'échec de la lutte clandestine, il reprend son métier d'ouvrier (p. 34). Le lien fictionnel est fort entre vie ouvrière, lutte politique, lutte armée. 
ouvrier c'est encore faire de la politique, même dans un moment de repli. La question qui se pose est celle de l'après, des raisons qui poussent à rester ouvrier quand les motivations politiques semblent avoir disparues ou, pour être plus précis, quand la défaite paraît acquise : après la grève de la Fiat à l'automne 1980. À n'en pas douter, cette grève qui échoua fut « la fin d'une époque ». Paul Ginsborg rapporte les paroles - épitaphe pour une génération ouvrière - prononcées, lors d'une assemblée générale des grévistes tenue le I5 octobre I980 au cinéma Smeraldo de Turin, par Giovanni Falcone, délégué de la section carrosserie de Mirafiori :

[...] un compagno, poche sere fa, mi diceva: "È un fatto storico, un altro compagno come noi, aveva parlato nel '69, oggi parli tu e si chiude un’epoca." Allora si apriva, ora si chiude... Mi lascia l'amaro in bocca questo. Perché per me dodici anni di lotta non sono stati soltanto dodici anni così, ma è stata una lunga esperienza politica, lo è stata per tutti. [...] Almeno ho la soddisfazione di aver chiuso in bellezza e sono contento di tutte le lotte che ho fatto, al di là che il padrone non mi riprenda più ${ }^{24}$.

"Almeno ho la soddisfazione di aver chiuso in bellezza... " Il y a bien la certitude de quelque chose qui se termine, qui se ferme... c'est précisément cette idée que quelque chose s'est fermé qu'Erri De Luca reprend à diverses reprises, dont une fois en utilisant le verbe "chiudere " : "Sono stato uno che ha passato il frattempo tra il '77 e il '9s a fare mestiere di operaio come può intenderlo chi simbarca marinaio: per tenersi al largo e alla larga del mondo che gli si è chiuso dietro ${ }^{25}$. "Un monde donc s'est fermé. Après, plus rien n’importe, même pas ce qui peut advenir de la vie personnelle : "Non mi è più importato un accidente di quello che mi è capitato dopo l'autunno dell'so ${ }^{26}$. " Seuls restent une fidélité, un souvenir qu'évoque le personnage d'Aceto arcobaleno:

In altri tempi insieme ad altri avevo vegliato davanti ai cancelli di una fabbrica per sbarrare all'alba l'ingresso, oppure nelle case vuote che avevamo preso, aspettando la truppa che sarebbe venuta a sgombrarci. Erano le notti dell'insonnia comune in cui uno nel freddo poteva sentire il proprio fiato salirsene in cielo dritto e caldo come un incenso acceso. In quell'autunno di Francia, Natale del nord, cosa stavo facendo? Reclamavo dei soldi, battendomi per averli e non li desideravo. Cosa mi legava a quegli uomini, oltre la necessità? Solo il ricordo del fiato di quelle altre notti ${ }^{27}$.

Un lien fait du souvenir d'un souffle qui monte au ciel comme un encens. La dimension spirituelle paraît indubitable. Il y a, dans la volonté




de maintenir ce lien en demeurant à la dernière place («sono rimasto a fare l'operaio comune, al rango più basso della gerarchia ${ }^{28}$ ") comme un destin que l'on ne peut qu'accepter : "Poi uno si trova a trent'anni senza studi e non sa fare altro e cosi quel mestiere si appiccica adosso e lo si fa perché quella è la porzione in terra che è toccata. "Cette phrase dit le destin (la part qui m'est échue ${ }^{29}$ ) mais aussi la nécessité : or la nécessité c'est un élément de l'analyse politique, ça relève de l'analyse concrète d'une situation, la conscience de la nécessité c'est ce qui peut inciter à agir, c'est même, pour Machiavel, ce qui peut rendre les hommes bons! Il y a là le signe d'une aporie possible, un risque de blocage : mais nous savons - Erri De Luca nous l'a dit - qu' existe une issue de secours, " en haut à gauche "... sur la page à lire et à écrire.

Fidélité à l'expérience de sa génération ayant vécu le communisme dans l'espace-temps libéré de quelques nuits partagées d'émeute et de résistance et volonté de trouver " les paroles d'accompagnement » de cette expérience dans la lecture nécessaire des textes sacrés : si l'on définit ainsi une des caractéristiques de l'écriture d'Erri De Luca, on accepte de dépasser ce qui peut apparaitre comme une contradiction, on inscrit son écriture dans une tension. On pourrait, mutatis mutandis, reprendre à son propos le jugement de Machiavel sur Laurent de Médicis : "si vedeva in lui essere due persone diverse, quasi con impossibile coniunzione congiunte ». Et il est vrai que dans l'œuvre d'Erri De Luca, l'intérêt pour l'empreinte du doigt de Dieu dans les vies et les textes et la fidélité à l'expérience révolutionnaire cœxistent. Cette cœxistence n'allait pas de soi car d'autres chemins étaient possibles et ont été (et sont) parcourus. Ainsi, en France, Benny Lévy, un des fondateurs d'un groupe radical des années 1968, la Gauche prolétarienne, avait, au terme d'un parcours de réflexion partant de cette expérience révolutionnaire, choisi définitivement la voie de l'étude religieuse et son dernier livre, Le Meurtre du pasteur. Critique de la vision politique $d u$ monde $e^{30}$, dénonçait l'impasse du chemin politique qu'il avait parcouru avec d'autres. À l'opposé, on pouvait tenter de lire les années 1968 en s'appuyant sur la méthode historique critique et ses instruments de lecture des archives et des textes ${ }^{31}$, sans estimer que seul le Livre peut donner du

28. Lettera da una città bruciata, op. cit., p. 47.

29. Notons qu'il pourrait s'agir d'une réminiscence biblique ; ainsi, pour donner un exemple, l'édition CEI rend Isä̈e, 57, 6 par une expression très proche : "Tra le pietre levigate del torrente è la parte che ti spetta / esse sono la porzione che ti è toccata."

30. B. Lévy, Le Meurtre du pasteur. Critique de la vision politique du monde, Paris, Grasset/Verdier, 2002.

3I. Je pense par exemple, pour l'Italie, au livre d'un autre ancien militant de Lotta continua qui fait l'histoire de la période des années I968: G. Crainz, Il paese mancato. Dal miracolo economico agli anni '8o, Roma, Donzelli, 2003. 
sens. Mais cette cœxistence " presque impossible» qu'Erri De Luca tente de maintenir a des effets sur sa propre lecture de son passé et de l'histoire de sa génération. Dans Nocciolo d'oliva, Erri De Luca fait parler Jésus qui déclare le caractère infructueux d'une révolution " quand elle est seulement politique " et prône une " révolte d'âmes en flammes, d'êtres sans défense passionnés de sainteté 32 ". Dans certains textes où il affirme sa solidarité avec ses vieux camarades, il fait passer au second plan l'analyse politique et historique pour insister sur l'empathie et l'amour ${ }^{33}$. La voix de la caritas, de l'amour à fondement divin, tend parfois à couvrir celle du révolté et on peut se demander s'il continuera à parler de Dieu à la troisième personne, en témoin indirect ou s'il sera à son tour "marqué d'une empreinte digitale ». Jusqu’à présent cette tension entre vision politique et religieuse de l'époque historique qu'il a vécue avec passion à vingt ans, lui a permis de trouver dans la littérature et l'étude des textes sacrés " une occupation qui ne semble pas privée de sens ».

32. Nocciolo d'oliva, p. 28 : "Non porta frutto la rivoluzione quando è solo politica. I deboli, i poveri, gli offesi devono armarsi d'altro. Solo una rivolta d'anime in fiamme, di inermi infervorati di santità può scalzare dai troni le molte Roma del mondo."

33. Lettera da una città bruciata, p. 76 : "Mi sono a lungo distolto dagli abbracci, ora non più." 